\title{
Hypertensive stretch regulates endothelial exocytosis of Weibel-Palade bodies through VEGF receptor 2 signaling pathways
}

Yan Xiong ${ }^{1}$, Zhenqian $\mathrm{Hu}^{1}$, Xiaofan $\mathrm{Han}^{1}$, Beibei Jiang ${ }^{1}$, Rongli Zhang ${ }^{2}$, Xiaoyu Zhang ${ }^{1}$, Yao Lu ${ }^{1}$, Chenyang Geng ${ }^{1}$, Wei $\mathrm{Li}^{3}$, Yulong $\mathrm{He}^{4}$, Yingqing Huo ${ }^{1}$, Masabumi Shibuya ${ }^{5}$, Jincai Luo ${ }^{1}$

${ }^{1}$ Laboratory of Vascular Biology, ${ }^{2}$ Non-human Primate Research Center, Institute of Molecular Medicine, Peking University, Beijing 100871, China; ${ }^{3}$ People's Hospital, Peking University, Beijing 100871, China; ${ }^{4}$ Laboratory of Vascular and Cancer Biology, Cyrus Tang Hematology Center, Soochow University, Suzhou, Jiangsu 215325, China; ${ }^{5}$ Institute of Physiology and Medicine, Jobu University, Takasaki, Japan

Regulated endothelial exocytosis of Weibel-Palade bodies (WPBs), the first stage in leukocyte trafficking, plays a pivotal role in inflammation and injury. Acute mechanical stretch has been closely associated with vascular inflammation, although the precise mechanism is unknown. Here, we show that hypertensive stretch regulates the exocytosis of WPBs of endothelial cells (ECs) through VEGF receptor 2 (VEGFR2) signaling pathways. Stretch triggers a rapid release (within minutes) of von Willebrand factor and interleukin-8 from WPBs in cultured human ECs, promoting the interaction between leukocytes and ECs through the translocation of P-selectin to the cell membrane. We further show that hypertensive stretch significantly induces P-selectin translocation of intact ECs and enhances leukocyte adhesion both ex vivo and in vivo. Stretch-induced endothelial exocytosis is mediated via a VEGFR2/PLC $\gamma 1 /$ calcium pathway. Interestingly, stretch also induces a negative feedback via a VEGFR2/Akt/nitric oxide pathway. Such dual effects are confirmed using pharmacological and genetic approaches in carotid artery segments, as well as in acute hypertensive mouse models. These studies reveal mechanical stretch as a potent agonist for endothelial exocytosis, which is modulated by VEGFR2 signaling. Thus, VEGFR2 signaling pathways may represent novel therapeutic targets in limiting hypertensive stretch-related inflammation.

Keywords: endothelial cells; stretch; VEGF receptor; exocytosis of Weibel-Palade bodies

Cell Research (2013) 23:820-834. doi:10.1038/cr.2013.56; published online 23 April 2013

\section{Introduction}

The endothelium is equipped with specific storage granules called Weibel-Palade bodies (WPBs), which store a variety of bioactive substances to rapidly respond to various chemical agonists, such as thrombin, histamine and VEGF [1]. The WPBs contain von Willebrand factor (vWF), t-PA, P-selectin, interleukin-8 (IL-8) and endothelin [2], which are involved in the regulation of injury, inflammation and thrombosis. Thus, the regulation of

Correspondence: Jincai Luo

Fax: +86-10-6275-0922

E-mail: jincailuo@pku.edu.cn

Received 9 September 2012; revised 14 January 2013; accepted 28 Februray 2013; published online 23 April 2013 endothelial exocytosis has been extensively studied. The regulation of WPB exocytosis involves the activation of canonical pathways mediated by second messengers, such as calcium and cAMP [3, 4], and the induction of a negative pathway executed by nitric oxide (NO), which inhibits the exocytosis of WPBs by the S-nitrosylation of $\mathrm{N}$-ethylmaleimide-sensitive factor (NSF), a component of the membrane fusion and exocytotic machinery [5, 6]. So far, however, the vast majority of our knowledge on the regulation of WPB exocytosis has been obtained from the studies conducted with chemical agonists, such as thrombin, histamine and VEGF. Little is known about the role of mechanical stimuli in this process. On the other hand, endothelial cells (ECs) are constantly exposed to pulsatile blood flow and pressure, which create shear stress and stretch stress, respectively. ECs are 
subjected to high, unidirectional laminar shear stress in straight arteries, whereas ECs at branch regions are exposed to disturbed flow. ECs under laminar shear stress are resistant to atherosclerotic risk, whereas ECs under disturbed flow is associated with inflammatory dysfunction and susceptibility to atherosclerotic injury $[7,8]$. It has been shown that a mechanosensory complex consisting of VEGF receptor 2 (VEGFR2), VE-cadherin and PECAM-1 at endothelial cell-cell junctions is required for flow-dependent cell alignment and inflammatory activation $[9,10]$. Like shear stress, stretch physiologically maintains endothelial homeostasis and vascular tone. An increase in stretch stress has also been linked to vascular inflammation, but the molecular mechanism remains elusive. Elevated blood pressure is positively associated with circulating proinflammatory and prothrombotic markers [11], including some WPB components such as endothelin-1 (ET-1) [12]. These observations suggest that the endothelial response to stretch stress may contribute to vascular inflammation. Consistent with this notion, excessive ventilatory strain on the lung quickly enhances the expression of intercellular adhesion molecule-1, vascular cell adhesion molecule-1 and P-selectin [13], which are essential adhesion molecules in mediating leukocyte recruitment during inflammation. Similarly, the inflammatory endothelial responses to mechanical stretch have also been reported in intra-arterial stent implantation, in which the local vascular wall is subjected to mechanical stretch $[14,15]$ and in grafted veins that are stretched by high arterial pressures [16]. These studies provide clues to cellular and molecular mechanisms by which mechanical stretch contributes to vascular inflammation.

We thus hypothesized that stretch induces endothelial exocytosis of WPBs and evaluated the effects of stretch on the exocytosis of WPBs in cultured human ECs and intact ECs of mouse carotid arteries. To keep our experimental system consistent, we chose the model of static biaxial stretch as the high flow rate for perfusing the vessels to create cyclic stretch generates a significant shear force that produces potential confounding effects [17].

\section{Results}

\section{Stretch triggers endothelial $v W F / W P B$ exocytosis}

To determine the effect of stretch on WPB exocytosis of cultured ECs, human umbilical vein ECs (HUVECs) were subjected to stretches of various magnitudes (120\%-150\% strain with the static control set at $100 \%$, Figure 1A), and the amount of vWF released into the medium was measured by ELISA. Upon stretch (150\%), HUVECs rapidly (within $5 \mathrm{~min}$ ) secreted a significant amount of vWF while their cell membrane remained in- tact (Supplementary information, Figure S1A and S1B). Stretch induced vWF secretion in a time- and magnitudedependent manner (Figure 1B and 1C), similar to the responses to chemical agonists, such as thrombin and VEGF $[18,19]$. When dissecting the time dependency of stretch-induced $\mathrm{vWF}$ release rate, it is interesting to note that $\mathrm{vWF}$ release rate decreased quickly after an initial burst of secretion within 5 min (Supplementary information, Figure S1B) and continued to decrease gradually thereafter (Supplementary information, Figure S1C), indicating the presence of a possible negative feedback pathway in the regulation of VWF release. Stretch also induced the release of IL- 8 and ET-1, two other components of WPBs, in a pattern similar, albeit slightly variant, to vWF release (Supplementary information, Figure S1D and S1E). Similar results were obtained from human arterial ECs (Supplementary information, Figure S1F). Immunofluorescence staining analysis revealed a significant translocation of P-selectin to the cell membrane in HUVECs exposed to stretch (Figure 1D). As P-selectin translocation initiates inflammation, we examined leukocyte adhesion to stretched ECs by using HL-60, a leukocyte cell line. The results indicated that increased translocation of P-selectin induced by stretch mediates leukocyte adhesion in cultured HUVECs, as the leukocyte adhesion can be dose-dependently inhibited by P-selectin-neutralizing antibody pretreatment (Figure $1 \mathrm{E}$ and $1 \mathrm{~F})$. This P-selectin-mediated pro-adhesive effect of stretch was confirmed in another leukocyte cell line, U937 (Supplementary information, Figure S2A and S2B).

To assess whether stretch promotes P-selectin translocation in intact ECs of vessels, isolated mouse carotid arteries were exposed to normal $(75 \mathrm{mmHg})$ or high intraluminal pressures $(150 \mathrm{mmHg}$, which dilated artery segments by $150 \%$ as compared with $75 \mathrm{mmHg}$, Figure 2A), and then harvested for immunohistochemical staining. As shown in Figure 2B, the treatment of high intraluminal pressure significantly increased the translocation of Pselectin to the luminal surface of vessels. Further studies indicated that increased translocation of P-selectin mediates leukocyte adhesion to intact ECs of artery fragments (Figure 2C).

\section{Stretch induces endothelial exocytosis mainly through VEGFR2}

Previous work demonstrated that VEGFR2 mediates vWF/WPB exocytosis induced by VEGF [19]. Therefore, we set out to delineate the roles of VEGFRs in stretchtriggered WPB exocytosis. The phosphorylation level of VEGFR2 was significantly increased by stretch, while that of VEGFR1 was barely detectable (Figure 3A). 
A

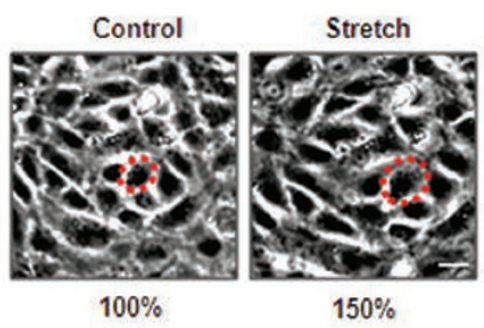

C

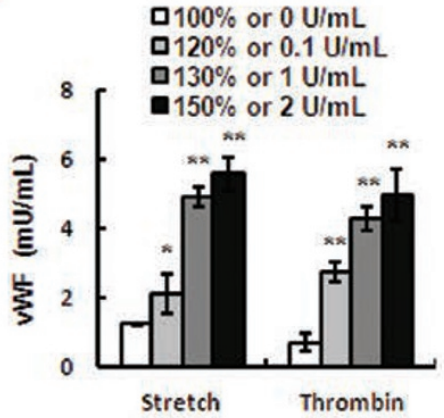

B

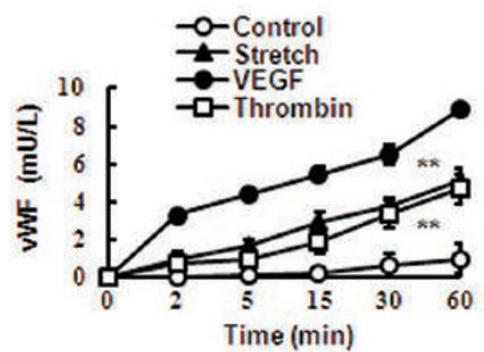

D

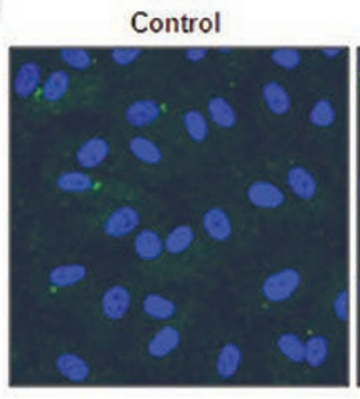

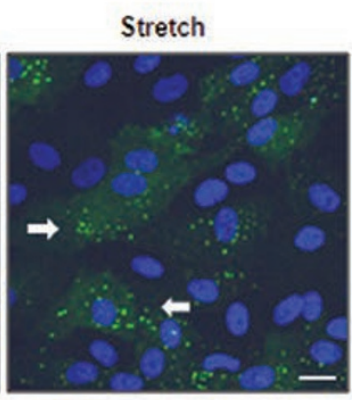

E
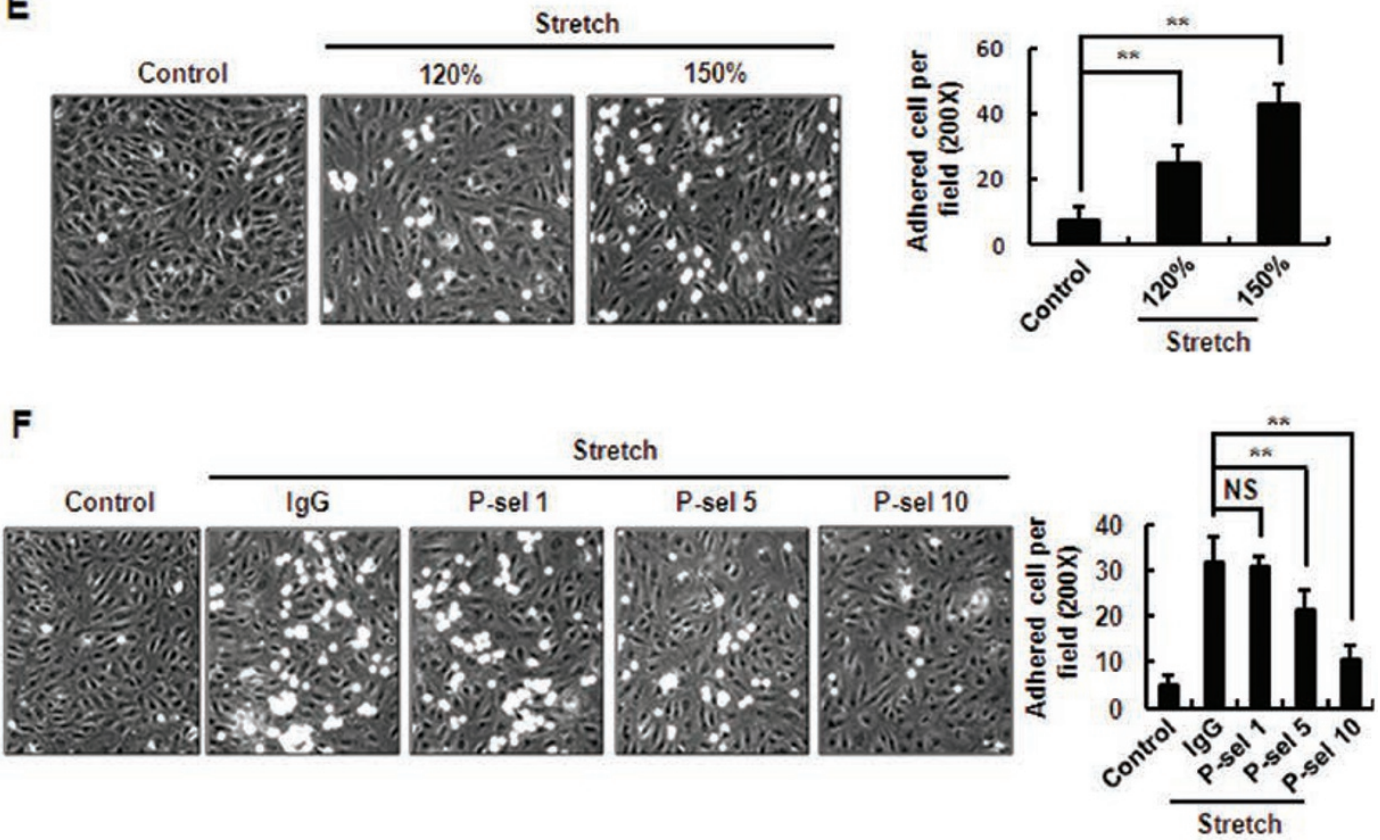

Figure 1 Stretch induces rapid WPB/VWF exocytosis from ECs. (A) The area of a single HUVEC was enlarged by $150 \%$ after static biaxial stretch compared with the non-stretched state (100\%). (B) Time course of vWF release. HUVECs were stretched $(150 \%)$ and treated with VEGF $(50 \mathrm{ng} / \mathrm{ml})$ or thrombin $(1 \mathrm{U} / \mathrm{ml})$ for the different time periods. The amount of vWF in the supernatant was measured by ELISA ( ${ }^{* *} P<0.01$, compared with control). (C) Dose dependence of vWF release. HUVECs were incubated with the indicated concentrations of thrombin, or subjected to different magnitudes of stretch for $1 \mathrm{~h}\left({ }^{*} P<0.05\right.$, ${ }^{* *} P<0.01$, compared with control). (D) Cell surface immunofluorescence staining of P-selectin in HUVECs. Solid arrows indicate P-selectin translocation after stretch. Scale bar: $10 \mu \mathrm{m}$. (E) HL-60 cell adhesion to HUVEC monolayers after different magnitudes of stretch $\left({ }^{* *} P<0.01\right.$, compared with control). (F) HL-60 cell adhesion to HUVEC monolayers after stretch, pretreated with different doses of P-selectin-neutralizing antibody (P-sel, $\mu \mathrm{g} / \mathrm{ml})$ or isotype control antibody $(\mathrm{lgG}, 10 \mu \mathrm{g} / \mathrm{ml})$ as indicated (control stands for non-pretreated cells, ${ }^{* *} P<0.01$, compared with $\lg G$ ). All the results are representative of three individual experiments and presented as mean $\pm \mathrm{SD}(n=4)$. 
A

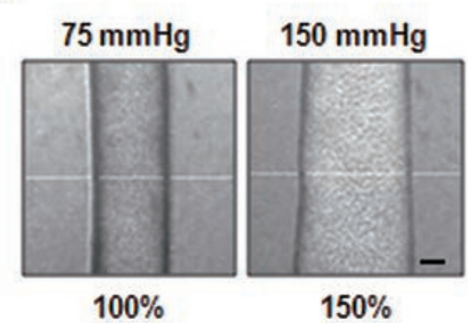

B

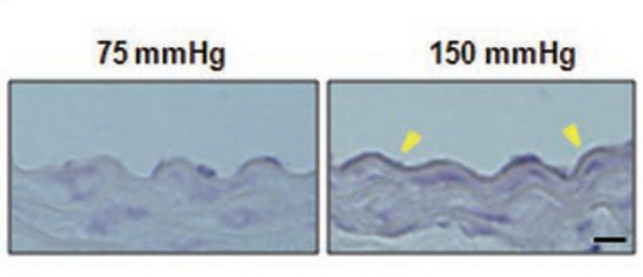

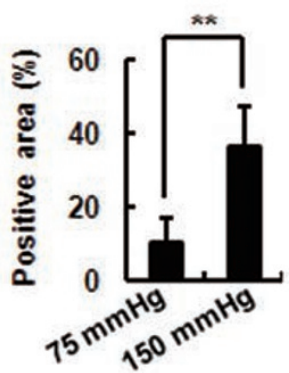
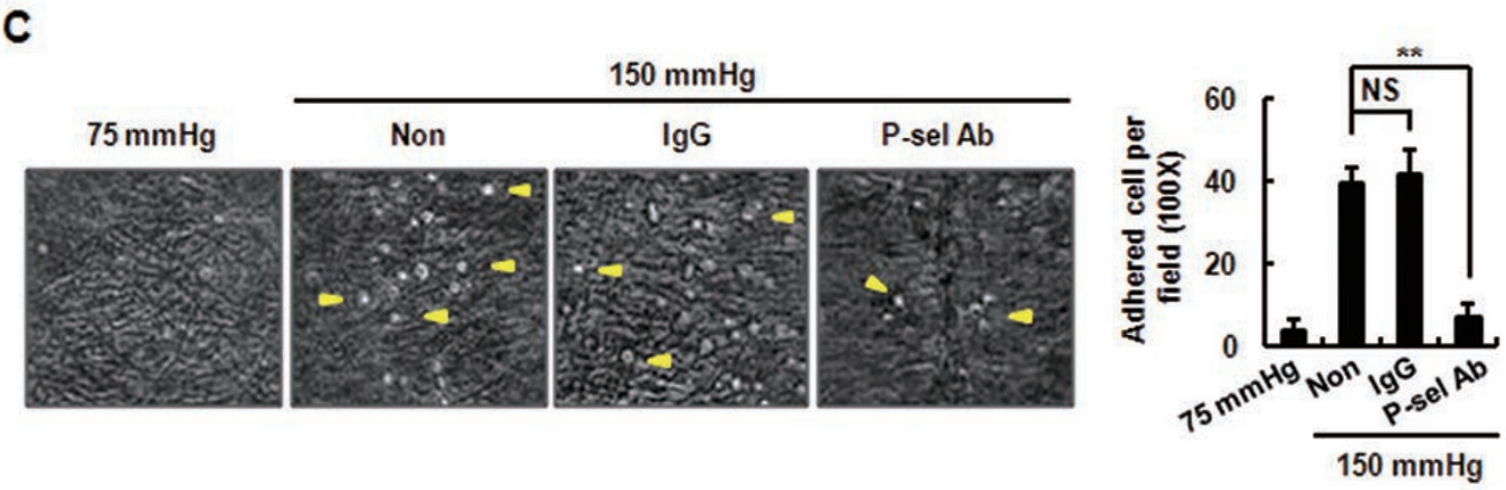

Figure 2 Stretch significantly induces P-selectin translocation and leukocyte adhesion. (A) The outer diameter of carotid artery segment exposed to $150 \mathrm{mmHg}$ pressure was dilated by $150 \%$, compared with $75 \mathrm{mmHg}(100 \%)$. Scale bar: $200 \mu \mathrm{m}$. (B) Left: immunohistochemical analysis of P-selectin expression on the intact endothelium of isolated carotid artery segments under $75 \mathrm{mmHg}$ or $150 \mathrm{mmHg}$ intraluminal pressure. Right: statistical analysis of the percentage of the positive staining area in the total surface area $\left({ }^{* *} P<0.01\right.$, compared with $\left.75 \mathrm{mmHg}\right)$. Scale bar: $10 \mu \mathrm{m}$. (C) Left: HL-60 cell adhesion to the lumen of carotid arteries exposed to $75 \mathrm{mmHg}$ or $150 \mathrm{mmHg}$ pressure, with or without pretreatment by lgG or P-selectin antibody (10 $\mu \mathrm{g} / \mathrm{ml}$ ). Right: quantitative analysis of HL-60 adhesion (non indicates non-pretreated cells, ${ }^{* *} P<0.01$, compared with nonpretreated cells). Results are representative of three individual experiments and presented as mean $\pm \operatorname{SD}(n=5)$.

We then assessed the effects of VEGFR1 and VEGFR2 knockdowns by specific short hairpin RNAs (shRNAs) on stretch-induced vWF exocytosis. VEGFR2 knockdown strikingly reduced vWF release, while VEGFR1 knockdown did not have much effect, confirming the predominant role of VEGFR2 (Figure 3B). Furthermore, to exclude the influence of VEGF auto-secretion during stretch, we pretreated HUVECs with a VEGF-neutralizing antibody. The antibody significantly inhibited VEGFinduced VEGFR2 tyrosine phosphorylation and endothelial exocytosis, but did not affect stretch-induced VEGFR2 phosphorylation (Figure 3C), vWF release (Figure $3 \mathrm{E}$ ), or leukocyte adhesion in (Figure 3F) ECs or intact vessels (Supplementary information, Figure S3), indicating that stretch alone activates VEGFR2, independently of ligand binding. This finding was further confirmed in VEGFR2-overexpressing 293A cells (Supplementary information, Figure S4A and S4B). In addition, inhibition of the Src kinase slightly, but significantly, attenuated the stretch-induced VEGFR2 activation (Figure 3D), indicating that stretch causes VEGFR2 activation at least in part through the Src kinase.

\section{A VEGFR2/PLC $1 / \mathrm{Ca}^{2+}$ pathway positively regulates} stretch-induced endothelial exocytosis

Our previous work showed that the activation of the VEGFR2/PLC $\gamma 1 / \mathrm{Ca}^{2+}$-signaling pathway plays a major role in mediating VEGF-induced vWF exocytosis [19]. Therefore, we further explored whether this pathway is activated by stretch. Indeed, PLC $\gamma 1$ phosphorylation at $\mathrm{Tyr}^{783}$ was substantially increased within 5 min after stretch and lasted at least $15 \mathrm{~min}$ (Figure 4A). A rapid increase of intracellular $\mathrm{Ca}^{2+}$ concentration was also detected (Figure 4B). The activation of the PLC $11 / \mathrm{Ca}^{2+}$ signaling pathway was greatly attenuated by VEGFR2 knockdown (Figure 4A and 4C). Furthermore, specific shRNA targeting PLC $\gamma 1$ greatly reduced stretch-induced $\mathrm{Ca}^{2+}$ accumulation and vWF release, as compared with 
A

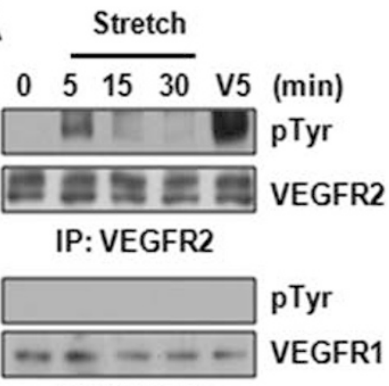

IP: VEGFR1
B

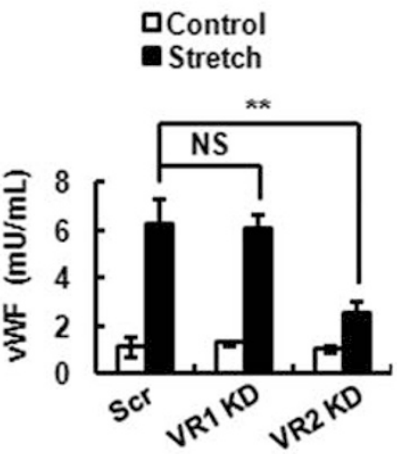

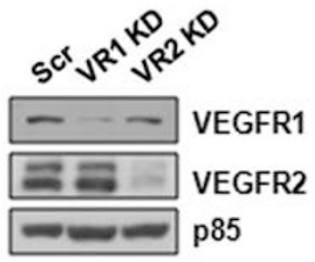

C

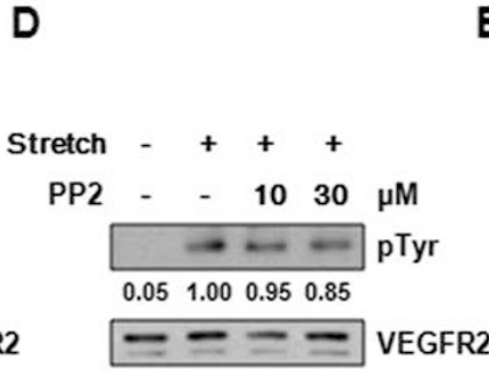

IP: VEGFR2

IP: VEGFR2

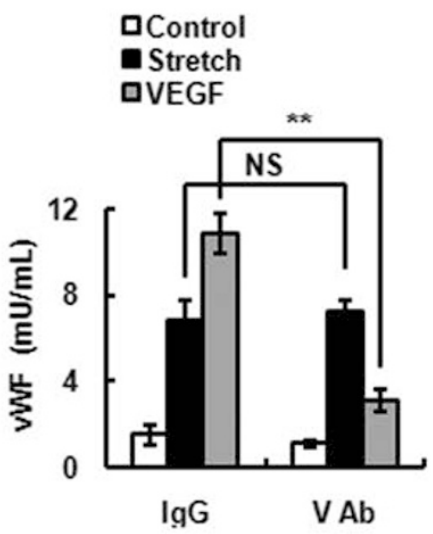

$\mathbf{F}$

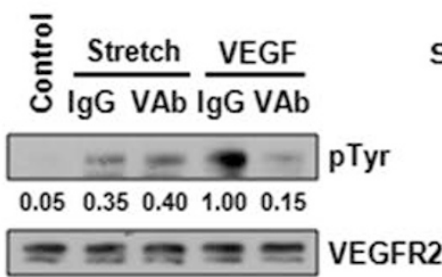

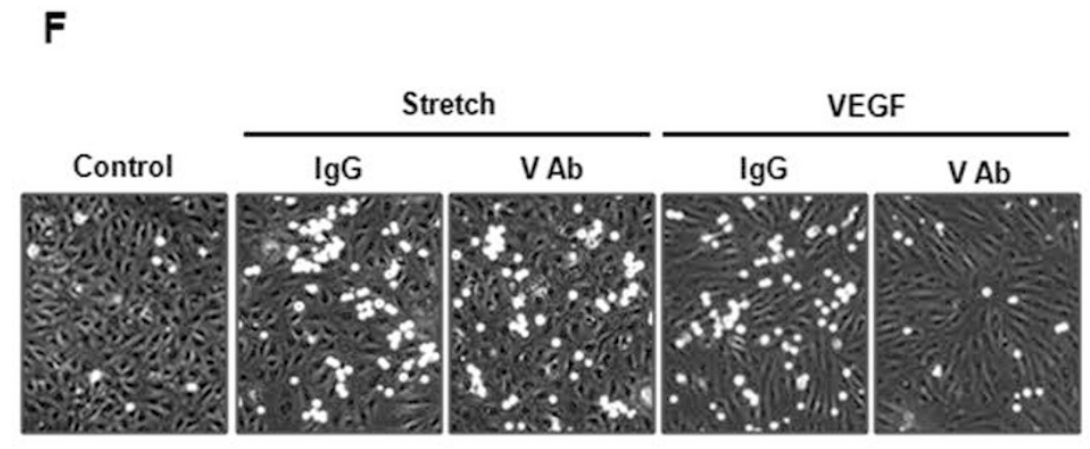

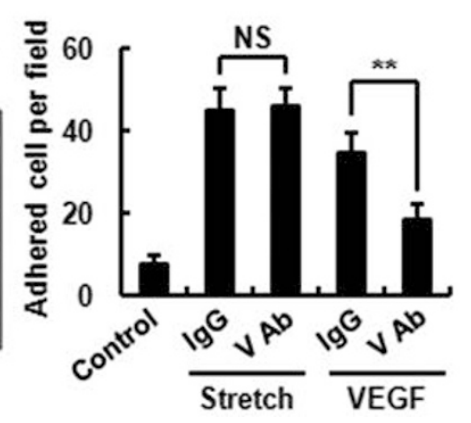

Figure 3 VEGFR2 mediates stretch-induced WPB exocytosis. (A) Immunoprecipitation and western blots of stretch- and VEGF (V5)-induced VEGFR1 and VEGFR2 activation at the indicated time points. (B) Left: vWF release in HUVECs expressing scrambled (Scr), VEGFR1 or VEGFR2 shRNAs (VR1 KD or VR2 KD. ${ }^{* *} P<0.01$, compared with scrambled. NS $=$ not significant). Right: western blots of the knockdown efficiency of shRNAs targeting VEGFR1 or VEGFR2 in HUVECs (VR1 KD or VR2 KD). (C) Western blots of stretch- and VEGF-induced VEGFR2 activation in the presence of $1 \mu \mathrm{g} / \mathrm{ml}$ isotype control (lgG) or VEGF-neutralizing antibody (V Ab). (D) Western blots of stretch-induced VEGFR2 activation in the presence of DMSO, 10 $\mu \mathrm{M}$ or $30 \mu \mathrm{M}$ PP2 (Src inhibitor). (E) vWF release in HUVECs pretreated with $1 \mu \mathrm{g} / \mathrm{ml} \mathrm{lgG}$ or $\mathrm{V}$ Ab, stimulated by stretch or VEGF $(50 \mathrm{ng} / \mathrm{ml})$ for $1 \mathrm{~h}\left({ }^{* *} P<0.01\right.$, compared with $\mathrm{lgG}$, NS = not significant). (F) Left: representative results of HL-60 cell adhesion to HUVEC monolayers pretreated with $1 \mu \mathrm{g} / \mathrm{ml} \mathrm{IgG} \mathrm{or} \mathrm{V} \mathrm{Ab,} \mathrm{and} \mathrm{stimulated} \mathrm{by} \mathrm{stretch} \mathrm{or} \mathrm{VEGF} \mathrm{(50} \mathrm{ng} / \mathrm{ml})$. Right: quantitative analysis of HL-60 cell adhesion ( ${ }^{* *} P<0.01$, compared with lgG. NS = not significant).

scrambled shRNA (Figure 4C and 4D). In accordance with these results, pretreatment of HUVECs with a $\mathrm{Ca}^{2+}$ chelator, BAPTA, also partially lowered stretch-induced
vWF release (Figure 4E). The above data clearly demonstrate the essential role of the $\mathrm{PLC} \gamma 1 / \mathrm{Ca}^{2+}$ pathway in regulating vWF exocytosis. 
A

Stretch

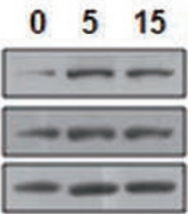

(min)

P.PLC

PLCy1

p85

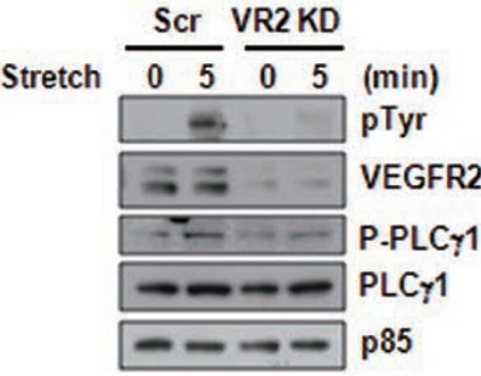

C
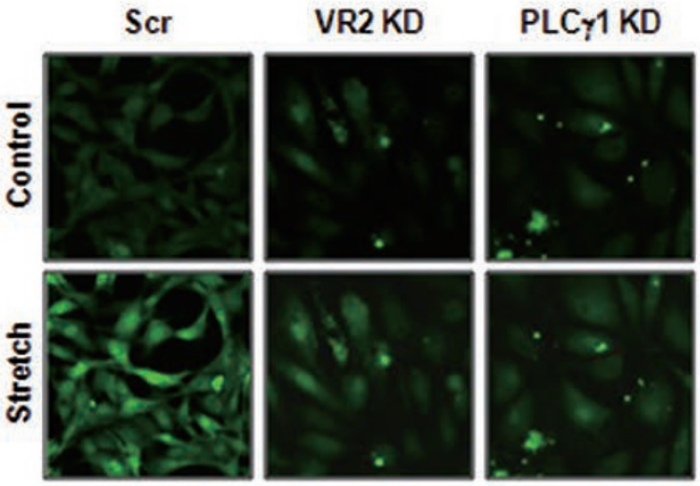

D

-Control

- Stretch

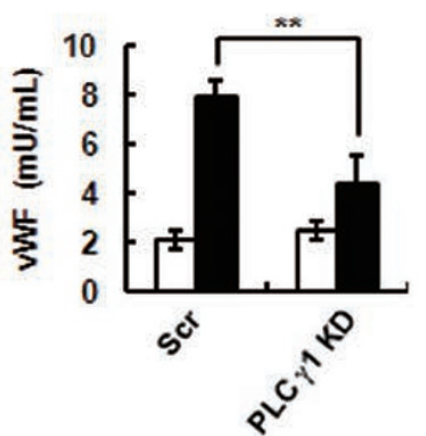

B
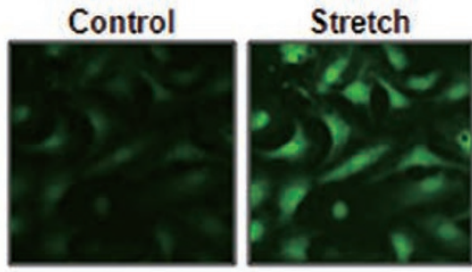

Stretch
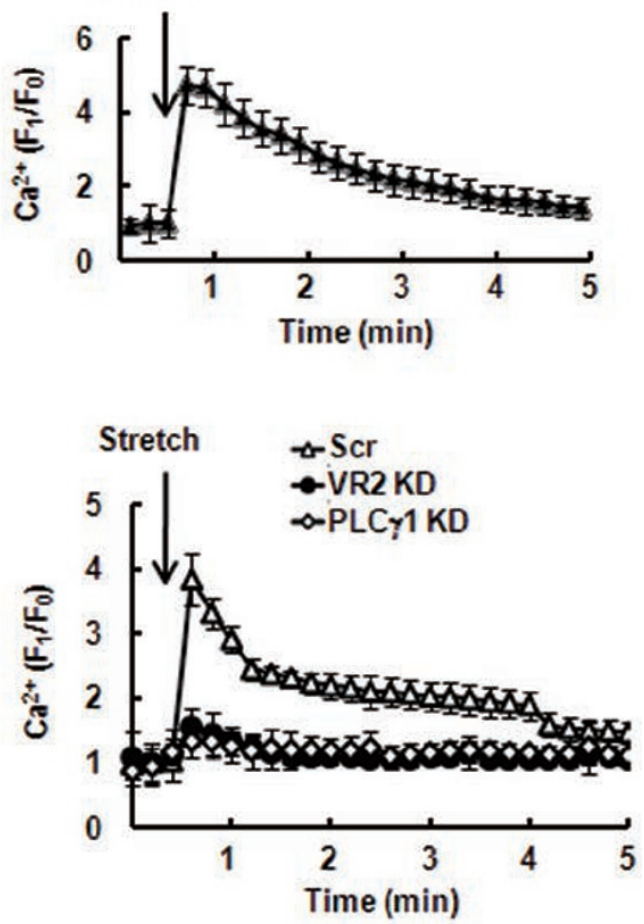

E

\section{口Control}

- Stretch

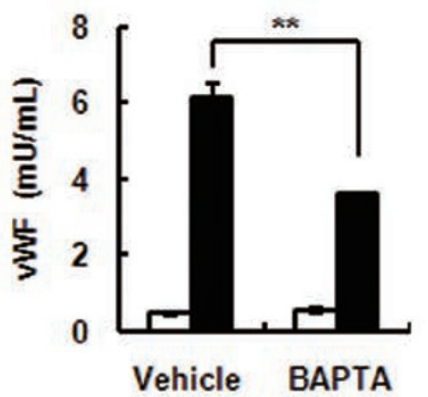

Figure $4 \mathrm{PLC} \gamma 1 / \mathrm{Ca}^{2+}$ pathway positively regulates stretch-induced vWF exocytosis. (A) Upper: phosphorylation of PLC $\gamma 1$ in HUVECs stretched for the indicated time periods. Lower: phosphorylation of PLC $\gamma 1$ in HUVECs expressing scrambled or VEGFR2-targeting shRNAs. (B) Upper: $\mathrm{Ca}^{2+}$ measurement in stretch-stimulated HUVECs. Lower: quantitative analysis of $\mathrm{Ca}^{2+}$ mobilization. (C) Left: $\mathrm{Ca}^{2+}$ measurements in stretch-stimulated HUVECs expressing scrambled, VEGFR2 or PLC $\gamma 1$ shRNAs (PLC $\gamma 1 \mathrm{KD}$ ). Right: quantitative analysis of $\mathrm{Ca}^{2+}$ mobilization. (D) Left: vWF release in HUVECs expressing scrambled or PLC $\gamma 1$ shRNAs $\left({ }^{* *} P<0.01\right.$, compared with scrambled). Right: western blots showing PLC $\gamma 1$ knockdown efficiency. (E) vWF release in HUVECs treated with $10 \mu \mathrm{M}$ BAPTA or DMSO (vehicle) before stretch $\left({ }^{* *} P<0.01\right.$, compared with DMSO vehicle). 
A VEGFR2/Akt/NO pathway negatively regulates stretchinduced endothelial exocytosis

VEGFR2 activates the Akt/endothelial nitric oxide synthase (eNOS) pathway, which promotes NO production and negatively regulates vWF exocytosis [18]. Therefore, we tested whether this pathway also plays a negative role under stretch stimulation. Stretch significantly increased the activations of Akt and eNOS as early as $5 \mathrm{~min}$ after stretch. These activations were attenuated in VEGFR2-knockdown HUVECs, indicating that stretch activates the Akt/eNOS pathway via VEGFR2 (Figure $5 \mathrm{~A})$. Furthermore, stretch increased NO production within a short time frame (Figure 5B), and markedly promoted the S-nitrosylation of NSF (Figure 5C) that negatively regulates exocytosis by preventing vesicle recycle. VEGFR2 knockdown also attenuated NO production and the S-nitrosylation of NSF (Figure 5B and 5C). These data indicate that VEGFR2-triggered eNOS/NO pathway may contribute to the decreased $\mathrm{vWF}$ release after the initial burst within $5 \mathrm{~min}$. To confirm the effect of $\mathrm{NO}$ on stretch-induced vWF exocytosis, we preincubated HUVECs with SNAP, an NO donor that provides exogenous NO (Supplementary information, Figure S5A), and found that SNAP pretreatment significantly impaired $\mathrm{vWF}$ exocytosis in a dose-dependent manner (Figure 5D). Consistently, the amount of vWF release was significantly increased in HUVECs expressing the shRNAs targeting Akt1 and Akt 2 or eNOS, compared with scrambled controls (Figure 5E). Furthermore, to confirm the effect of Akt/eNOS-signaling blockade on stretch-induced vWF release, we pretreated HUVECs with LY-294002 that inhibits PI3K, the Akt upstream activator or L-NAME that specifically inhibits eNOS (Supplementary information, Figure S5B), and found that contrary to the effect of SNAP, these treatments dose-dependently increased vWF release (Figure 5F), indicating the negative role of the Akt/eNOS pathway. Thus, we concluded that stretch instantly triggers both PLC $\gamma 1 / \mathrm{Ca}^{2+}$ and Akt/eNOS signaling pathways, and finely regulates endothelial exocytosis through the interplay between these two pathways (Supplementary information, Figure 6). Consistently, stretch also induced PLC $\gamma 1 / \mathrm{Ca}^{2+}$ and Akt signaling pathways in VEGFR2-overexpressing 293A cells (Supplementary information, Figure S4C and S4D).

Stretch-induced WPB exocytosis is mediated through VEGFR2 signaling pathways in mouse carotid arteries

We further examined the role of VEGFR2 signaling pathways in stretch-stimulated mouse carotid arteries. Immunohistological studies showed that high intraluminal pressure triggered $\mathrm{vWF}$ release and translocation of P-selectin to the endothelial surface, which was par- tially blocked by the pretreatment of the vessels with the VEGFR2 inhibitor SU5416 or the $\mathrm{Ca}^{2+}$ chelator BAPTA (Figure 6A and Supplementary information, Figure S7A). These results suggest that high pressure-induced $\mathrm{P}$-selectin translocation and VWF release is dependent on VEGFR2/ $\mathrm{Ca}^{2+}$ signaling. To determine the role of eNOS in stretch-induced WPB exocytosis, the carotid arteries from eNOS-knockout $\left(\mathrm{eNOS}^{-/}\right.$) or wild-type (WT) mice were isolated and exposed to high or normal intraluminal pressure. Immunohistological analysis demonstrated that the expression level of VWF and P-selectin on the endothelial surface of $\mathrm{eNOS}^{-/-}$mice was significantly higher than that of the WT mice, under both the high and normal intraluminal pressures (Figure 6B and Supplementary information, Figure S7B). Consistently, the level of stretch-induced leukocyte adhesion in carotid arteries was significantly higher in $\mathrm{eNOS}^{-/}$mice than in WT mice (Figure 6C). These results suggest that VEGFR2 modulates stretch-induced exocytosis in intact ECs of vessels.

\section{Involvement of stretch-induced endothelial exocytosis in acute hypertensive mouse models}

To study the potential role of stretch-induced endothelial exocytosis in acute hypertension in vivo, we used a portal hypertension mouse model in which the blood pressure is elevated mainly due to mechanical factor from partial portal vein ligation (PPVL) [20, 21]. As shown in Figure 7A, PPVL operation significantly enhanced portal vein pressure, which was suppressed by the pretreatment of Urapidil hydrochloride, a blood pressure lowering drug [22] (Figure 7A). Immunohistological staining and intravital studies indicated that the PPVL significantly increased P-selectin translocation to the luminal surface and leukocyte adhesion to the endothelial layer, which were inhibited by the pretreatment of Urapidil hydrochloride (Figure 7B and 7C). Urapidil hydrochloride per se has no effect on stretch-induced endothelial exocytosis in ECs (data not shown). Taken together, the above data suggest that PPVL-induced endothelial exocytosis is dependent on vascular wall stretch by blood pressure.

To investigate the positive and negative regulation pathways of stretch-induced endothelial exocytosis in acute hypertensive mouse models, VEGFR2 inhibition and $\mathrm{eNOS}^{-/-}$mice were employed in PPVL model. Immunohistological staining indicated that the inhibitor of VEGFR2 markedly reduced the increased P-selectin translocation to the luminal surface induced by the increased pressure in mesenteric veins (Figure 8A). Pselectin translocation was further enhanced in the mesenteric veins of $\mathrm{eNOS}^{-/-}$mice after PPVL procedure (Figure 
A
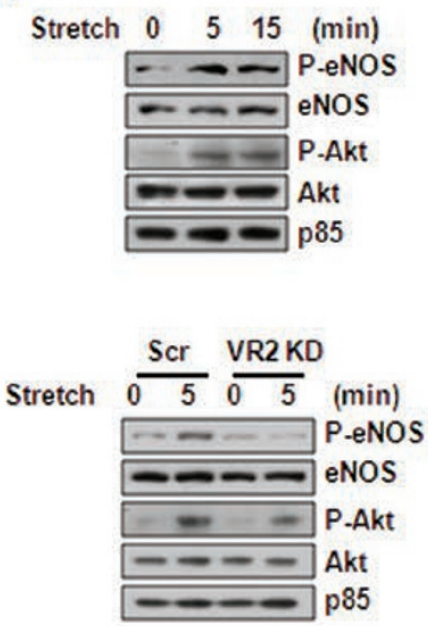

C

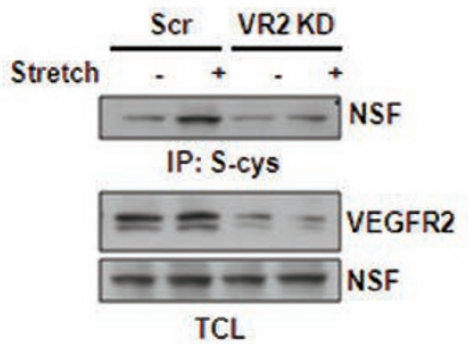

E

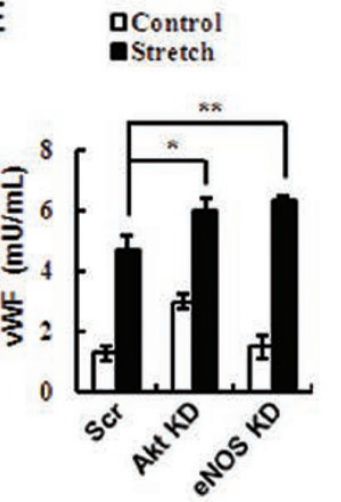

$\mathrm{B}$
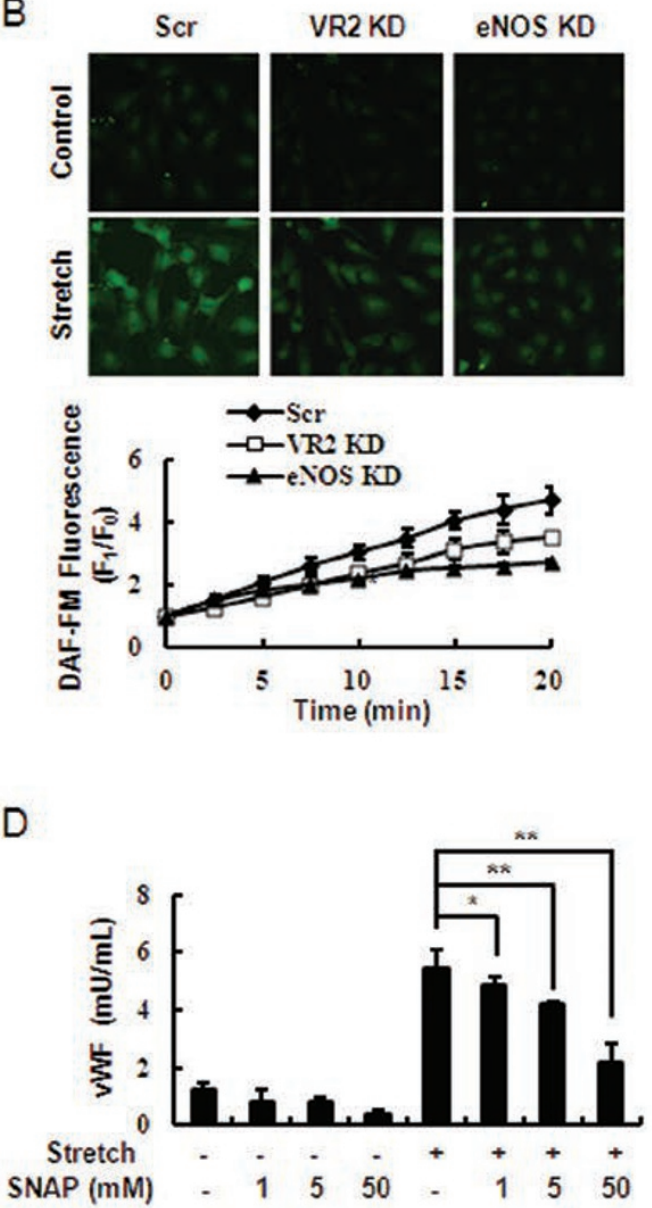

Figure $5 \mathrm{Akt/NO/NSF}$ pathway negatively regulates stretch-induced vWF exocytosis. (A) Upper: phosphorylation of Akt and eNOS in HUVECs stretched for the indicated time periods. Lower: phosphorylation of Akt and eNOS in HUVECs expressing scrambled or VEGFR2-targeting shRNAs. (B) Upper: stretch-induced NO release in HUVECs expressing scrambled, VEGFR2 or eNOS-targeting shRNAs (eNOS KD). Lower: quantitative analysis of NO release. (C) Western blots of stretch-induced NSF nitrosylation. HUVECs were stretched and harvested at the indicated time points, the lysates were precipitated with S-nitrosylated cysteine antibody and blotted with NSF antibody. (D) Stretch-induced VWF release in HUVECs pretreated with different doses of SNAP as indicated for $4 \mathrm{~h}\left({ }^{*} P<0.05,{ }^{* *} P<0.01\right.$, compared with DMSO-pretreated control). (E) Left: VWF release in HUVECs expressing scrambled, eNOS or Akt1 and 2 shRNAs (eNOS KD or Akt KD, ${ }^{*} P<0.05$, ${ }^{* *} P<0.01$, compared with scrambled). Right: western blots of eNOS (upper) and Akt (lower) knockdown efficiency. (F) Stretch-induced VWF release in HUVECs pretreated with different doses of LY 294002 or L-NAME as indicated for $1 \mathrm{~h}\left({ }^{*} P<0.05,{ }^{* *} P<0.01\right.$, compared with DMSO-pretreated control). 
A

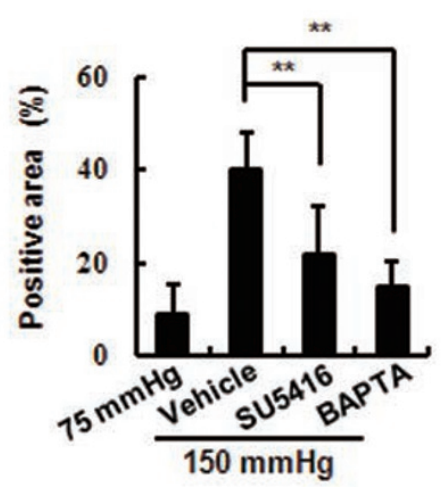

B

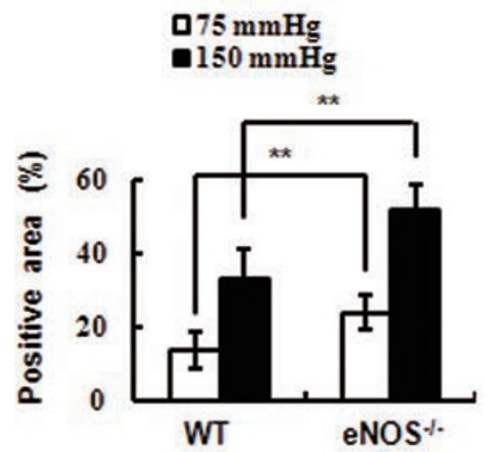

C

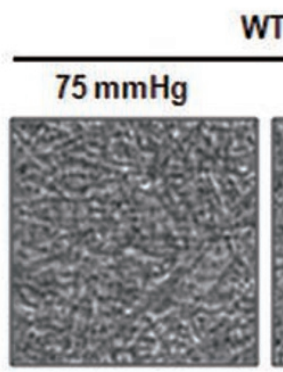

WT

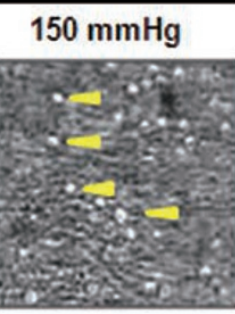

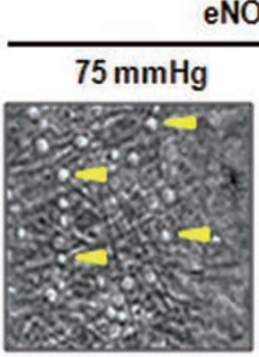

eNOS $\%$

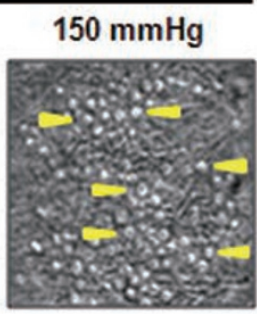

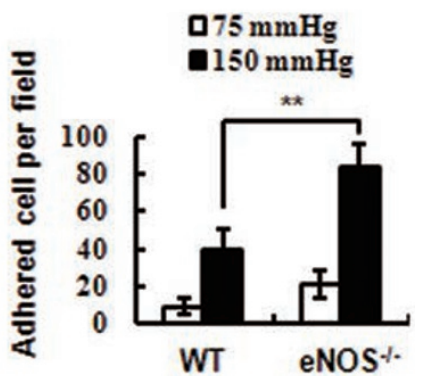

Figure 6 Stretch induces P-selectin translocation and leukocyte adhesion in mouse carotid arteries. (A) Quantitative analysis of the immunohistochemical staining of isolated carotid arteries exposed to $75 \mathrm{mmHg}$ or $150 \mathrm{mmHg}$ and pretreated with 10 $\mu \mathrm{M}$ SU5416, $10 \mu \mathrm{M}$ BAPTA or DMSO (vehicle) by calculating the percentage of P-selectin-positive area in total surface area ( ${ }^{* \star} P$ $<0.01$, compared with the vehicle). (B) Quantitative analysis of the immunohistochemical staining of carotid arteries isolated from WT or eNOS ${ }^{-/-}$mice exposed to $75 \mathrm{mmHg}$ or $150 \mathrm{mmHg}$ by calculating the percentage of P-selectin-positive area in total surface area $\left({ }^{* *} P<0.01\right.$, compared with WT). (C) Left: representative photomicrographs showing HL-60 cells adherent to the lumen of carotid arteries that were isolated from WT or eNOS ${ }^{-1-}$ mice and were exposed to $150 \mathrm{mmHg}$ or $75 \mathrm{mmHg}$ pressure. Arrowheads indicate adhered cells. Right: quantitative analysis of HL-60 cell adhesion $\left({ }^{* *} P<0.01\right.$, compared with WT mice).

8B). Intravital analysis also demonstrated that leukocyte adhesion to the endothelial layer was significantly increased after PPVL procedure, which was suppressed by P-selectin-neutralizing antibody but not by control antibody (Figure 8C). Consistent with the above findings, the level of leukocyte adhesion in $\mathrm{eNOS}^{-/}$mice was also slightly higher than that in WT mice, and the difference was amplified by the increase of vein pressure after PPVL procedure (Figure 8D).

\section{Discussion}

The results presented here show that acute stretch rapidly triggers the exocytosis of WPBs from cultured human arterial and venous ECs, and intact ECs of carotid mouse arteries, promoting leukocyte adhesion via P-se- lectin translocation. Moreover, we identified VEGFR2 as a key modulator for the exocytosis of WPBs through two signaling pathways: one is a positive pathway primarily mediated by PLC $\gamma 1 / \mathrm{Ca}^{2+}$ signaling, while the other is a negative feedback pathway mediated by Akt/NO/NSF signaling. It is still unclear about the mechanism of possible cross talk between the two pathways. It appears that PLC $\gamma 1$ activation is quick, strong, but transient, while Akt activation is relatively delayed but long-lasting, and eNOS activation is relatively weak but long-lasting as well (Supplementary information, Figure S6B). In addition, PLC $\gamma 1$ knockdown blocked eNOS activation, but did not impair Akt phosphorylation (Supplementary information, Figure $\mathrm{S} 6 \mathrm{C}$ ). We thus propose that in response to stretch, $\mathrm{PLC} \gamma 1 / \mathrm{Ca}^{2+}$ signaling initiates endothelial exocytosis, but it soon triggers a long-lasting activation 
A

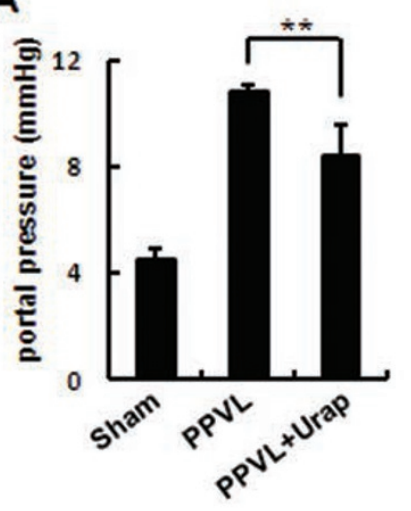

B

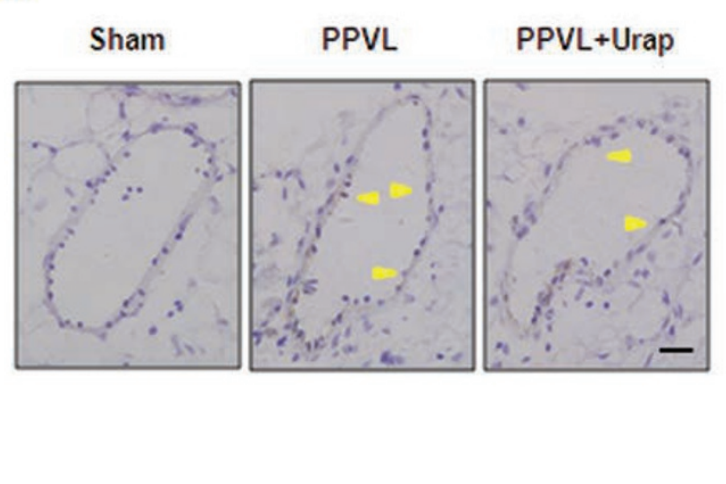

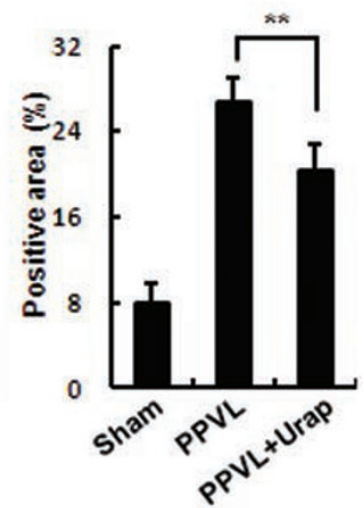

\section{C}
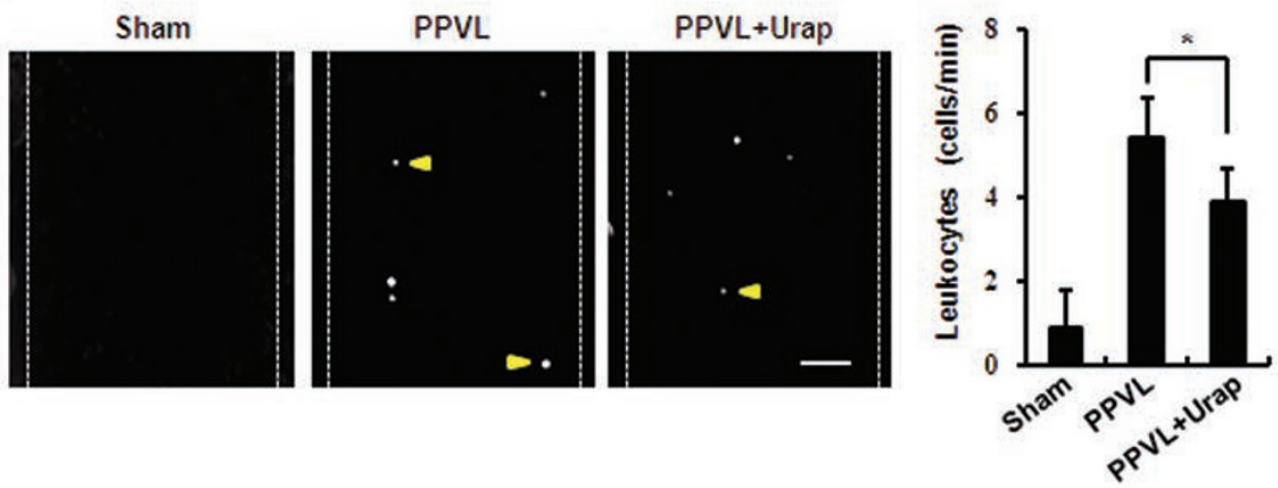

Figure 7 Blood pressure affects P-selectin translocation and leukocyte adhesion in acute hypertensive mesenteric veins. (A) Mesurement of portal pressure (PP) in mice after sham or PPVL procedure. Some PPVL mice were pretreated with Urapidil hydrochloride $(55 \mathrm{mg} / \mathrm{kg}$ ) as indicated. (B) Representative P-selectin staining in mesenteric veins of mice after sham or PPVL procedure. Some PPVL mice were pretreated with Urapidil hydrochloride. Arrowheads indicate P-selectin-positive areas. Scale bar: $10 \mu \mathrm{m}$. Quantification was performed by calculating the percentage of P-selectin-positive area in total surface area ( ${ }^{*} P<0.01$, compared with PPVL, right panel). (C) Intravital analysis of leukocyte adhesion to endothelial cells in mesenteric veins of mice after sham or PPVL procedure. Some PPVL mice were pretreated with Urapidil hydrochloride. Arrowheads indicate adhered leukocytes. Scale bar: $50 \mu \mathrm{m}$. Quantification was performed by counting adhered cell number per field per minute ( ${ }^{*} P<0.05$, compared with PPVL, right panel).

of eNOS that serves as a negative regulator of exocytosis.

Previously, laminar shear stress, another mechanical stimulus for ECs, was shown to induce vWF release [23], but its biological significance and underlying signaling mechanism largely remained unclear. The results of the present study show that stretch stress rapidly activates ECs to release the proinflammatory and prothrombotic substances, such as vWF, P-selectin, ET-1 and IL-8 from WPBs, promoting leukocyte rolling and adhesion (Figure 1 and Supplementary information, Figure S2). Importantly, we show that hypertensive stretch quickly stimulates endothelial exocytosis and leads to leukocyte adhesion in acute hypertension mouse models, which further deterio- rates in hypertensive eNOS ${ }^{-/}$mice. Thus, our study provides another mechanistic explanation at the cellular and molecular levels for the close link between stretch stress and vascular inflammation, and thrombosis in hypertension.

Previously, it was shown that shear stress activated VEGFR2 via conformational change in a ligand-independent manner [24]. The present work indicates that stretch may activate VEGFR2 through similar mechanism based on the following lines of evidence: (1) the VEGF-neutralizing antibody did not affect stretch-induced VEGFR2 phosphorylation (Figure 3C); (2) the VEGF-neutralizing antibody failed to block stretch-induced WPB exocytosis and leukocyte adhesion in cultured ECs (Figure 3E and 
A
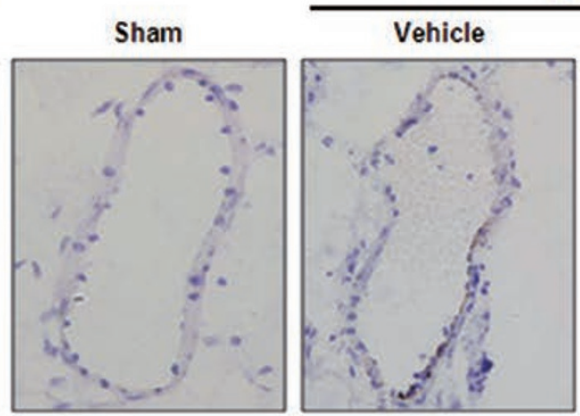

PPVL

B

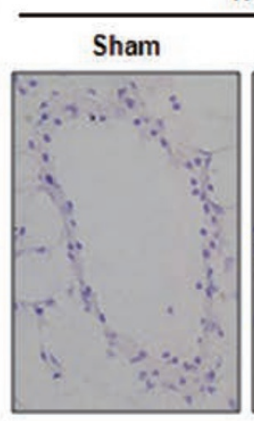

WT
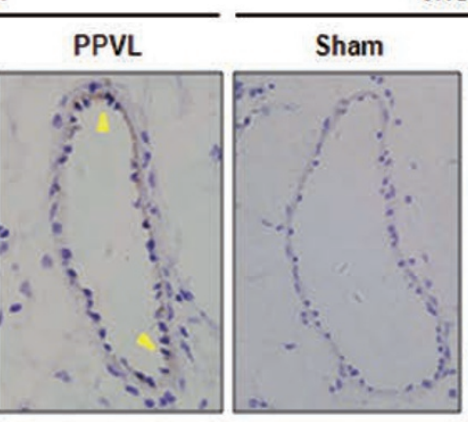

eNOS $\%$
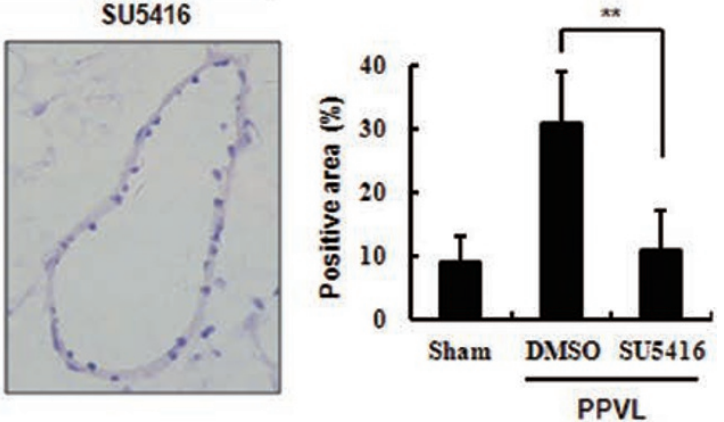

C
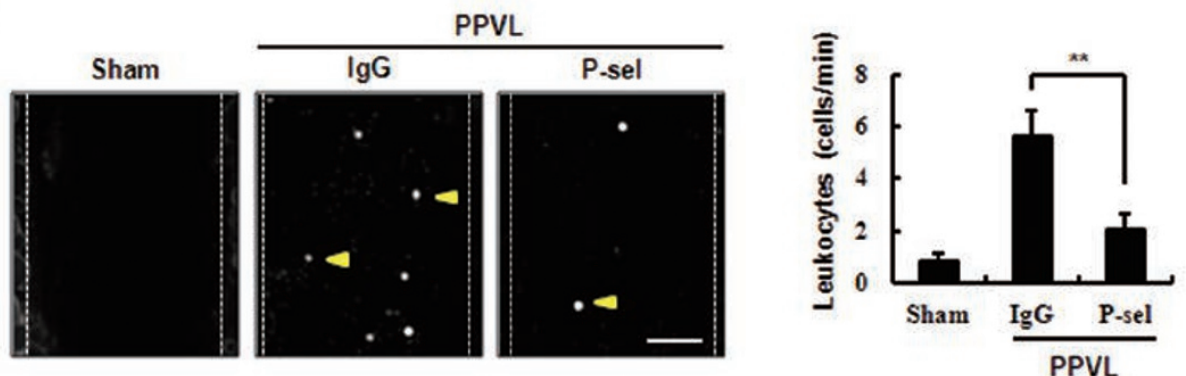

D
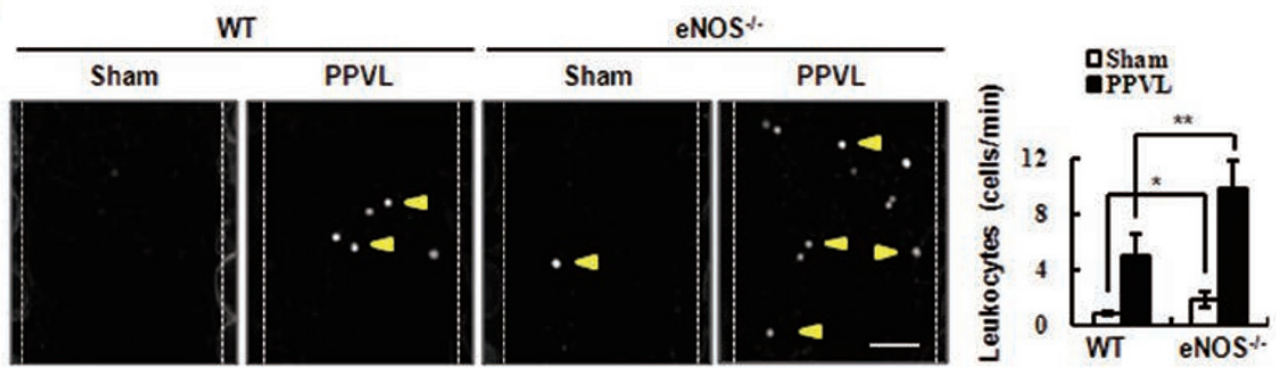

Figure 8 Stretch induces P-selectin translocation and leukocyte adhesion in acute hypertensive mesenteric veins. (A, B) Representative P-selectin staining of mesenteric veins in mice after sham or PPVL procedure. Quantification was performed by calculating the percentage of $\mathrm{P}$-selectin-positive area in total surface area $\left({ }^{\star} P<0.05\right.$, ${ }^{* *} P<0.01$, right panel). (A) Mice were intravenously injected with DMSO (vehicle) or $10 \mu \mathrm{M}$ SU5416 before sham or PPVL procedure. (B) Comparison of P-selectin stainings between WT and $\mathrm{eNOS}^{-/-}$mice after sham or PPVL procedure. Arrowheads indicate P-selectin-positive area. Scale bar: $10 \mu \mathrm{m}$. (C, D) Intravital analysis of leukocyte adhesion to endothelial cells in mesenteric veins in mice after sham or PPVL procedure. Arrowheads indicate adhered leukocytes. Scale bar: $50 \mu \mathrm{m}$. Quantification was performed by counting adhered cell number per field per minute $\left({ }^{*} P<0.05\right.$, ${ }^{* \star} P<0.01$, right panel). (C) Mice were intravenously injected with $10 \mu \mathrm{g} /$ $\mathrm{ml}$ P-sel antibody or IgG before sham or PPVL procedure. (D) WT or eNOS ${ }^{-/-}$mice were subjected to sham or PPVL procedure. 
3F), as well as intact ECs of vessels (Supplementary information, Figure S3); (3) mechanical stretch activated VEGFR2 that was exogenously expressed in 293A cells and its downstream signaling pathways (Supplementary information, Figure S4), similar to the case of the activation of angiotensin II type 1 receptor by stretch [25]. Other components of the mechanical sensor complex and downstream signaling cascades, e.g., stretchactivated cation channels, intergrin signaling and $\mathrm{G}$ protein-coupled receptor signaling known to be involved in the mechanotransduction in ECs $[9,26]$, may also play important roles in stretch-induced exocytosis of WPBs, which await further investigations.

As a vasodilator, eNOS-produced NO exerts beneficial effects on EC integrity, such as inhibition of leukocyte adhesion and platelet aggregation [27, 28]. Using pharmacological and genetic approaches, we showed that NO signaling plays a negative role in stretch-induced exocytosis (Figure 5D, 5E and 5F). Stretch-induced NO nitrosylates NSF (Figure 5C), which inhibits exocytosis [18]. Thus, NO signaling may play an important role as a negative feedback in the regulation of stretch-induced exocytosis and vascular inflammation.

The present study implicates VEGFR2 as the key linker between stretch stress and vascular inflammation, and thrombosis through regulating endothelial exocytosis of WPBs. VEGFR2 is expressed in both venous and arterial ECs. In addition, recent studies, including ours, have shown that VEGF induces WPB exocytosis in both arterial and venous ECs primarily mediated by VEGFR2 $[18,19,29]$. Therefore, it is not unexpected that stretch exerts a proexocytosis effect on both arteries and veins. The present study suggests that VEGFR2 may play critical roles in hypertensive stretch-induced inflammation caused by portal vein hypertension (Figure 8 ) and acute arterial hypertension (data not shown). In addition, our observation may also have important clinical implications for understanding pathological process of venous graft diseases. Autologous saphenous veins are widely used to circumvent occluded coronary arteries during coronary artery bypass grafting procedures in order to treat myocardial ischemic diseases [30]. Venous graft is susceptible to accelerated atherosclerosis and narrowing of the vessel lumen. Exposure to higher arterial pressure may stimulate endothelial exocytosis of grafted vein and lead to inflammation and thrombosis, the early processes of venous graft diseases [31]. This insight may provide a new therapeutic target for preventing venous graft failure.

It should be noted that there are several limitations in this study. First, as our observations were made only in acute phase of static stretch, the effects of long-term and cyclic stretch need to be further studied. Second, in this study, the levels of static stretch and static pressure employed in our in vitro and ex vivo models were relatively high. Therefore, cautions should be taken when interpreting the findings and the implications of this study. Third, the effects observed in our in vivo hypertension models may also be contributed by other factors, such as blood flow and vascular remodeling, which occur as vascular responses to long-term systemic hypertension.

In conclusion, our results reveal acute mechanical stretch as a potent agonist for endothelial exocytosis of WPBs, which is modulated by VEGFR2-signaling pathways. Our study provides new mechanistic insights into mechanical stretch stress-related vascular inflammation, thrombosis and other hypertension implications, and suggests possible use of VEGFR2-signaling pathways as novel therapeutic targets in inhibiting stretch-related inflammation.

\section{Materials and Methods}

\section{Reagents}

Recombinant human VEGF165 (VEGF), BAPTA-AM, fluo 4-AM, DAF-FM diacetate, lipofectamine 2000 and IL-8 ELISA kit were from Invitrogen (Carlsbad, CA, USA). Heparin, 4', 6-diamidino-2 phenylindole dihydrochloride (DAPI), polyclonal rabbit antibody against S-nitrosylated cysteine and dimethyl sulfoxide (DMSO) were from Sigma (St Louis, MO, USA). L-NAME was from Alexis (San Diego, CA, USA). SU5416 was from Merck and PP2 was from ENZO. Urapidil hydrochloride was from $\mathrm{Ny}$ comed Deutschland GmbH. Rabbit polyclonal antibodies to NSF, PLC $\gamma 1$, Akt and phosphotyrosine were from Santa Cruz Biotechnology (Santa Cruz, CA, USA). Mouse monoclonal antibodies to phospho-eNOS (Ser ${ }^{1177}$ ) (for detecting eNOS activation) and eNOS, rabbit polyclonal antibodies to phospho-PLC $\gamma 1\left(\mathrm{Tyr}^{783}\right)$ (for detecting PLC $\gamma 1$ activation), rat anti-mouse P-selectin and isotype control antibody were from BD Biosciences (San Diego, CA, USA). Mouse monoclonal antibodies to human P-selectin and VEGF, and ET-1 ELISA kit were from R\&D systems (Minneapolis, MN, USA). Rabbit polyclonal antibodies to phospho-PKC substrate and phospho-Akt ( $\mathrm{Ser}^{473}$ ) (for detecting Akt activation) were from Cell Signaling Technology (Beverly, MA, USA). Rabbit antisera against p85, VEGFR1 and VEGFR2 were obtained as previously described [32]. In the signaling experiments, p85 was mostly used as the loading control, as we found that general loading controls such as GAPDH, tubulin and actin were too abundant to accurately reflect protein loading in lysates. The vWF ELISA kit was described previously [19].

\section{Animals}

eNOS-knockout mice (C57BL/6J background, from Jackson Laboratory) were interbred with WT C57BL/6J mice (from Vital River Ltd Co) to generate eNOS-knockout and WT control littermate mice. For the comparison experiments, age- and gendermatched 8-10-week-old eNOS-knockout mice and their WT control littermates were used as indicated in the description of 
experimental procedures. All animal procedures were carried out according to the rules of Association for Assessment and Accreditation of Laboratory Animal Care International, and were approved by the Institutional Animal Care and Use Committee of Peking University.

\section{RNA interference}

To silence VEGF1, VEGFR2, PLC $\gamma 1$, eNOS and Akt1/2, we used a commercial lentiviral system from Sigma to deliver shRNAs. The target and control scrambled sequences are selected according to an open program (http://jura.wi.mit.edu/bioc/ siRNAext/). The targeting sequences for shRNAs are: PLC $\gamma 1$, 5'-GTGTGCAGTCAAAGCCCTCTT-3'; VEGFR1, 5'-GGCGACGAATTGACCAAAGCA-3'; VEGFR2, 5'-CTGACTTGGCCTCGG TCATTT-3'; eNOS, 5'-GTGGCCAACGCCGTGAAGATC-3'; Akt, 5'-GGAGGGTTGGCTGCACAAATT-3'; Akt2, 5'-CTCCTTGGCAAGGGAACCTTT-3'; control scrambled shRNA, 5'-CCTAAGGTTAAGTCGCCCTCG-3'.

\section{Cell culture}

HUVECs were isolated and cultured at $37{ }^{\circ} \mathrm{C}$ in a humidified atmosphere of $5 \% \mathrm{CO}_{2}$ as previously described [19]. Human arterial ECs were grown in endothelial growth medium-2-MV (EGM2-MV BulletKit, Clonetics, MD, USA). Cells were used from passages 3 to 6. 293A cells were obtained from ATCC and cultured in Dulbecco's modified Eagle's medium (DMEM) containing 10\% fetal bovine serum (FBS).

\section{Biaxial stretch of cultured cells}

Monolayers of ECs were subjected to an established device for the study of static biaxial stretch, which has been described in detail [33]. Serum-starved ECs were cultured on rat-tail collagencoated silicone elastic membrane (Specialty MFG, MI, USA) in a single-well device and were uniformly stretched by vertical indentation, resulting in sustained homogeneous strains of $120 \%-150 \%$ (static control as 100\%).

\section{ELISA analysis}

Confluent ECs were starved in serum-free M199 medium supplemented with $2 \%$ BSA for $4 \mathrm{~h}$, and then stimulated by stretch or other factors as indicated for different time periods and intensities. The supernatant was harvested and centrifuged at $3600 \mathrm{rpm}$ at $4{ }^{\circ} \mathrm{C}$. The concentration of vWF, IL- 8 and ET- 1 were assessed by the standard sandwich ELISA procedure according to the manufacturer's instructions.

\section{Virus preparation and infection}

Preparations of lentiviruses and retrovirus were made in 293T cells. $293 \mathrm{~T}$ cells were transfected, and after $48 \mathrm{~h}$ the virus containing supernatants was harvested. Three milliliters of the supernatants mixed with $3 \mathrm{ml}$ fresh M199 medium containing a final concentration of $8 \mu \mathrm{g} / \mathrm{ml}$ polybrene were used for infection of ECs, as previously described [19]. The medium was replaced with normal growth M199 medium after 24 h, and HUVECs were harvested for experiments after $72 \mathrm{~h}$ of infection.

\section{Western blot and immunoprecipitation analyses}

Confluent ECs were serum-starved for $16 \mathrm{~h}$ before stimulation. The stimulated cells were washed twice in ice-cold PBS and lysed in buffer containing $50 \mathrm{mM}$ HEPES, $150 \mathrm{mM} \mathrm{NaCl}, 1 \%$ Triton X-100, 10\% glycerol, $1.5 \mathrm{mM} \mathrm{MgCl}_{2}, 1 \mathrm{mM}$ EGTA, $5 \mathrm{mM}$ EDTA, $0.27 \mathrm{~g} / \mathrm{ml} \mathrm{Na}_{4} \mathrm{P}_{2} \mathrm{O}_{7}, 5 \mu \mathrm{g} / \mathrm{ml}$ aprotinin, $1 \mu \mathrm{g} / \mathrm{ml}$ Prostatin A, $1 \mu \mathrm{g} / \mathrm{ml}$ Antipan, $10 \mu \mathrm{g} / \mathrm{ml}$ Leupeptin, $1 \mathrm{mg} / \mathrm{ml}$ PMSF, $2 \mathrm{mM}$ $\beta$-glycerol phosphate, $10 \mathrm{mM} \mathrm{NaF}$ and $2 \mathrm{mM} \mathrm{Na}_{3} \mathrm{VO}_{4}$. The lysates were fractionated on $8 \%$ SDS-PAGE, followed by standard western blot analysis. For immunoprecipitation, $500 \mu 1$ lysates were precipitated with antibodies against $8 \mu$ l VEGFR1, VEGFR2 or Snitrosylated cysteine at $4{ }^{\circ} \mathrm{C}$ for $7 \mathrm{~h}$, incubated with $20 \mu \mathrm{l}$ Protein A beads for an additional $2 \mathrm{~h}$, denatured and then fractionated on 8\% SDS-PAGE and blotted with anti-phosphotyrosine, VEGFR1, VEGFR2 or NSF antibody.

\section{Measurement of intracellular free calcium}

Intracellular free calcium was measured by using the indicator dye fluo-4 AM. ECs were plated onto collagen-coated coverslips and incubated with fluo-4 AM in M199 containing 2\% BSA for 20 min at $37{ }^{\circ} \mathrm{C}$. After $2 \mathrm{~h}$ of starvation with M199 containing $2 \%$ BSA, cells were washed with PBS, stimulated by stretch, recorded by $40 \times$ oil objective lenses and analyzed with laser scanning confocal microscopy (Zeiss, Germany).

\section{Measurement of NO production}

Confluent ECs were starved in serum-free M199 medium supplemented with $2 \%$ BSA for $3 \mathrm{~h}$, and incubated with $5 \mu \mathrm{M}$ DAFFM diacetate in phenol red-free DMEM for 30 min at $37^{\circ} \mathrm{C}$. Then the cells were washed gently with PBS, stimulated by stretch or other factors as indicated for different time periods and intensities. The real-time changes of DAF fluorescence was recorded by $40 \times$ oil objective lenses and analyzed with laser scanning confocal microscopy (Zeiss, Germany). The specificity of this assay has been confirmed by the treatment of NO donors, inhibitors and knockdown analysis of eNOS shRNAs.

\section{In vitro leukocyte adhesion assay}

Confluent ECs were starved in serum-free M199 medium supplemented with $2 \%$ BSA for $4 \mathrm{~h}$, and then stimulated by stretch for $30 \mathrm{~min}$. Then the supernatant was discarded and $2 \times 10^{6} \mathrm{HL}-60$ or U937 cells were added to the surface of the ECs. After $30 \mathrm{~min}$ of incubation, the unbound cells were washed off three times with M199 medium, the bound cells were fixed in 4\% PFA and photomicrographs were taken on a Nikon inverted microscope with $20 \times$ objective lenses. In some experiments, P-selectin-neutralizing or control antibodies were added to the perfusion buffer $30 \mathrm{~min}$ before injection of HL-60 cells. The numbers of bound HL-60 cells in every single field were counted and analyzed by one-way ANOVA. Representative results of three independent experiments were shown.

\section{Immunofluorescence staining}

Confluent ECs were washed twice with cold PBS, fixed with $4 \%$ PFA supplemented with $0.2 \mathrm{~mol} / 1$ sucrose for $30 \mathrm{~min}$ at $4{ }^{\circ} \mathrm{C}$ and blocked with $5 \%$ goat serum for $30 \mathrm{~min}$ at room temperature (RT). After washing three times with PBS containing $0.2 \%$ Triton $\mathrm{X}-100$, the cells were incubated with an antibody to P-selectin or control antibody ( $1 \mu \mathrm{g} / \mathrm{ml}, \mathrm{R} \& \mathrm{D}$ systems, MN, USA) for $1 \mathrm{~h}$ at RT, washed another three times, then incubated with goat anti-mouse HRP-conjugated secondary antibody (Invitrogen, CA, USA) for $1 \mathrm{~h}$. Finally, the cells were counter-stained with DAPI and sealed 
with nail polish. Photomicrographs were taken by $40 \times$ oil objective lenses and analyzed with laser scanning confocal microscopy (Zeiss, Germany).

\section{Vessel stretch and ex vivo leukocyte adhesion assays}

Left and right common carotid arteries were isolated from 10-week-old male WT and eNOS-knockout mice, and immersed in an organ culture bath filled with M199 supplemented with $1 \%$ BSA for $2 \mathrm{~h}$. Each arterial segment was cannulated at both ends, and connected to an airtight perfusion circuit consisting of a threeport reservoir, a peristaltic syringe pump (Harvard Apparatus, MA, USA), and a pressure sensor to monitor the intraluminal pressure. After washing with phosphate saline buffer for $15 \mathrm{~min}$, the intraluminal pressure was set to $75 \mathrm{mmHg}$ or $150 \mathrm{mmHg}$ and maintained for $30 \mathrm{~min}$. To avoid effects of shear forces and cyclic stretch, the flow rate for vessel perfusion was set at low level $(0.37 \mathrm{ml} / \mathrm{min})$. For leukocyte adhesion assay, $5 \times 10^{6} / \mathrm{ml} \mathrm{HL}-60$ leukemic cells were injected into the lumen of the vessel and allowed to interact for another $30 \mathrm{~min}$. Finally, unbound cells were washed out in phosphate buffer for $10 \mathrm{~min}$ at $75 \mathrm{mmHg}$ and fixed with $4 \%$ PFA before taking photomicrographs on a Nikon inverted microscope with $20 \times$ objective lenses. In some experiments, SU5416, BAPTA, VEGF and P-selectin-neutralizing, or control antibodies were added to the perfusion buffer $30 \mathrm{~min}$ before the injection of HL60 cells. The numbers of bound cells in every single field were counted and analyzed by one-way ANOVA.

\section{Portal pressure measurement}

Measurement of portal pressure (PP) was performed as described previously [20]. A catheter from the portal vein was connected to a highly sensitive pressure transducer (SP 844, MEMSCAP, Norway). The external zero reference point was placed at the midportion of the animal. PP was measured by inserting a saline-filled 30-gauge microcannula (BD) into the portal vein toward liver. The insertion was achieved by a one-time penetration with no blood leak or suture ligation. The microcannula was connected to the pressure transducer through a short length of PE-10 tubing, and the pressure was digitalized by PowerLab data acquisition system (AD Instruments, Australia). All readings were performed in triplicate, monitored, and saved on a computer.

\section{PPVL procedure and intravital microscopy}

Male WT and eNOS-knockout mice of 8-10-week-old were analyzed for mesenteric hypertension induced by upstream PPVL procedure [20]. After anesthetizing with $70 \mathrm{mg} / \mathrm{kg}$ sodium pentobarbital, a midline abdominal incision was made, and the portal vein was freed from surrounding tissue. A ligature (5-0 suture) was placed around a 25-gauge blunt-tipped needle lying along the portal vein. Subsequent removal of the needle caused stenosis of the portal vein and varicosity of downstream mesenteric veins. In sham-operated mice, similar procedure was performed, but after the portal vein was isolated, no ligature was placed. The abdomen was then closed with 5-0 suture. To decrease blood pressure, Urapidil hydrochloride $(55 \mathrm{mg} / \mathrm{kg})$ was injected into tail vein of mice before PPVL. Immunohistological and intravital studies were performed $2 \mathrm{~h}$ after operation. For intravital microscopy, $0.1 \mathrm{mg} /$ $\mathrm{ml}$ Rhodamine 6G was injected to label leukocytes [34]. Adhered leukocytes were counted if they remained stationary for more than $20 \mathrm{~s}$. We counted the number of leukocyte adhering in the artery per microscope field $(100 \times)$ and presented the results as the number of leukocytes observed per field per minute.

\section{Immunohistochemistry}

The isolated carotid arteries were collected following intraluminal high pressure treatment, snap-frozen in OCT compound (Sakura, Tokyo, Japan), cut at $8 \mu \mathrm{m}$, and stained for immunohistochemistry using a rat anti-mouse P-selectin monoclonal antibody or isotype control antibody (BD Biosciences) according to standard procedures. The positive-staining areas in the intact vessel endothelium of each segment were outlined and quantified as the percentage of total area with ImagePro software [35].

\section{Statistical analysis}

Results are expressed as mean \pm SEM or SD on the basis of triplicate experiments. Statistical analysis was done using one-way ANOVA. A value of $P<0.05$ was considered statistically significant.

\section{Acknowledgments}

We thank Drs Quan Chen, Mian Long, Chunyang Xiong and Zhihong Li for helpful discussions on the project. We thank Drs Peace Cheng, Nanping Wang, Xiaocheng Gu and Iain C Bruce for reading the manuscript and offering valuable comments. We also thank Dr Xiuqin Zhang and Ms Ning Hou for technical assistance. Jincai Luo is supported by grants from the National Basic Research Program of China (2012CB945100) and the National Natural Science Foundation of China (81170098 and 81070115). Yulong He is supported by the Priority Academic Program Development of Jiangsu Higher Education Institutions.

\section{References}

1 Rondaij MG, Bierings R, Kragt A, van Mourik JA, Voorberg J. Dynamics and plasticity of Weibel-Palade bodies in endothelial cells. Arterioscler Thromb Vasc Biol 2006; 26:1002-1007.

2 Lowenstein CJ, Morrell CN, Yamakuchi M. Regulation of Weibel-Palade body exocytosis. Trends Cardiovasc Med 2005; 15:302-308.

3 Tranquille N, Emeis JJ. On the role of calcium in the acute release of tissue-type plasminogen activator and von Willebrand factor from the rat perfused hindleg region. Thromb Haemost 1991; 66:479-483.

4 Vischer UM, Wollheim CB. Epinephrine induces von Willebrand factor release from cultured endothelial cells: involvement of cyclic AMP-dependent signalling in exocytosis. Thromb Haemost 1997;77:1182-1188.

5 Jilma B, Pernerstorfer T, Dirnberger E, et al. Effects of histamine and nitric oxide synthase inhibition on plasma levels of von Willebrand factor antigen. J Lab Clin Med 1998; 131:151-156.

6 Matsushita K, Morrell CN, Cambien B, et al. Nitric oxide regulates exocytosis by S-nitrosylation of N-ethylmaleimidesensitive factor. Cell 2003; 115:139-150.

7 Chiu JJ, Chien S. Effects of disturbed flow on vascular endothelium: pathophysiological basis and clinical perspectives. Physiol Rev 2011; 91:327-387.

8 Traub O, Berk BC. Laminar shear stress: mechanisms by 
which endothelial cells transduce an atheroprotective force. Arterioscler Thromb Vasc Biol 1998; 18:677-685.

9 Tzima E, Irani-Tehrani M, Kiosses WB, et al. A mechanosensory complex that mediates the endothelial cell response to fluid shear stress. Nature 2005; 437:426-431.

10 Shay-Salit A, Shushy M, Wolfovitz E, et al. VEGF receptor 2 and the adherens junction as a mechanical transducer in vascular endothelial cells. Proc Natl Acad Sci USA 2002; 99:9462-9467.

11 Blann AD, Naqvi T, Waite M, McCollum CN. von Willebrand factor and endothelial damage in hypertension. J Hum Hypertens 1993; 7:107-111.

12 Lüscher TF, Seo B, Bühler FR. Potential role of endothelin in hypertension. Hypertension 1993; 21:752-757.

13 Miyao N, Suzuki Y, Takeshita K, et al. Various adhesion molecules impair microvascular leukocyte kinetics in ventilatorinduced lung injury. Am J Physiol Lung Cell Mol Physiol 2006; 290:L1059-L1068.

14 Gyöngyösi M, Yang P, Khorsand A, Glogar D. Longitudinal straightening effect of stents is an additional predictor for major adverse cardiac events: Austrian Wiktor Stent Study Group and European Paragon Stent Investigators. J Am Coll Cardiol 2000; 35:1580-1589.

15 Xia ZY, Yang H, Qu HQ, Cheng WD, Wang LX. Expression of P-selectin, von Willebrand and endothelin-1 after carotid artery stenting. Vasa 2011; 40:199-204.

16 Conte MS, Mann MJ, Simosa HF, et al. Genetic interventions for vein bypass graft disease: a review. J Vasc Surg 2002; 36:1040-1052.

17 Riou S, Mees B, Esposito B, et al. High pressure promotes monocyte adhesion to the vascular wall. Circ Res 2007; 100:1226-1233.

18 Matsushita K, Yamakuchi M, Morrell CN, et al. Vascular endothelial growth factor regulation of Weibel-Palade-body exocytosis. Blood 2005; 105:207-214.

19 Xiong Y, Huo YQ, Chen C, et al. Vascular endothelial growth factor (VEGF) receptor-2 tyrosine 1175 signaling controls VEGF-induced von Willebrand factor release from endothelial cells via phospholipase C- $\gamma 1$ - and protein kinase A-dependent pathways. J Biol Chem 2009; 284:23217-23224.

20 Iwakiri Y, Cadelina G, Sessa WC, Groszmann RJ. Mice with targeted deletion of eNOS develop hyperdynamic circulation associated with portal hypertension. Am J Physiol Gastrointest Liver Physiol 2002; 283:G1074-G1081.

21 Abraldes JG, Pasarín M, García-Pagán JC. Animal models of portal hypertension. World J Gastroenterol 2006; 12:65776584.

22 Dabiré H, Lacolley P, Chaouche-Teyara K, Fournier B, Safar ME. Relationship between arterial distensibility and low- frequency power spectrum of blood pressure in spontaneously hypertensive rats. J Cardiovasc Pharmacol 2002; 39:98-106.

23 Galbusera M, Zoja C, Donadelli R, et al. Fluid shear stress modulates von Willebrand factor release from human vascular endothelium. Blood 1997; 90:1558-1564.

24 Jin ZG, Ueba H, Tanimoto T, Lungu AO, Frame MD, Berk BC. Ligand-independent activation of vascular endothelial growth factor receptor 2 by fluid shear stress regulates activation of endothelial nitric oxide synthase. Circ Res 2003; 93:354-363.

25 Zou Y, Akazawa H, Qin Y, et al. Mechanical stress activates angiotensin II type 1 receptor without the involvement of angiotensin II. Nat Cell Biol 2004; 6:499-506.

26 Inoue R, Jensen LJ, Shi J, et al. Transient receptor potential channels in cardiovascular function and disease. Circ Res 2006; 99:119-131.

27 Napoli C, Ignarro LJ. Nitric oxide and pathogenic mechanisms involved in the development of vascular diseases. Arch Pharm Res 2009; 32:1103-1108.

28 Dudzinski DM, Michel T. Life history of eNOS: partners and pathways. Cardiovasc Res 2007; 75:247-260.

29 Lorenzi O, Frieden M, Villemin P, Fournier M, Foti M, Vischer UM. Protein kinase C-delta mediates von Willebrand factor secretion from endothelial cells in response to vascular endothelial growth factor (VEGF) but not histamine. $J$ Thromb Haemost 2008; 6:1962-1969.

30 Favaloro RG, Effler DB, Groves LK, Sheldon WC, Riahi M. Direct myocardial revascularization with saphenous vein autograft: clinical experience in 100 cases. Chest 1969; 56:279283.

31 Song H, Mowbray AL, Sykes MC, Jo H. Emerging role of IGF-1R in stretch-induced neointimal hyperplasia in venous grafts. Arterioscler Thromb Vasc Biol 2007; 27:1679-1681.

32 Lu Y, Xiong Y, Huo Y, et al. Grb-2-associated binder 1 (Gab1) regulates postnatal ischemic and VEGF-induced angiogenesis through the protein kinase A-endothelial NOS pathway. Proc Natl Acad Sci USA 2011; 108:2957-2962.

33 Liao XD, Wang XH, Jin HJ, Chen LY, Chen Q. Mechanical stretch induces mitochondria-dependent apoptosis in neonatal rat cardiomyocytes and $\mathrm{G} 2 / \mathrm{M}$ accumulation in cardiac fibroblasts. Cell Res 2004; 14:16-26.

34 Kobayashi T, Tahara Y, Matsumoto M, et al. Roles of thromboxane A (2) and prostacyclin in the development of atherosclerosis in apoE-deficient mice. J Clin Invest 2004; 114:784794.

35 Sa Q, Hoover-Plow JL. EMILIN2 (Elastin microfibril interface located protein), potential modifier of thrombosis. Thrombosis J 2011; 9:9-16.

(Supplementary information is linked to the online version of the paper on the Cell Research website.) 